\title{
Space weather effects on the low latitude D-region ionosphere during solar minimum
}

\author{
Abhikesh Kumar ${ }^{*}$ and Sushil Kumar
}

\begin{abstract}
The effects of the solar flares and the geomagnetic storms (disturbance storm time (Dst) $<-50 \mathrm{nT}$ ) during December 2006 to 2008, a period during the unprecedented solar minimum of solar cycles 23 and 24, have been examined on sub-ionospheric very low frequency (VLF) signals from NWC (19.8 kHz), NPM (21.4 kHz), VTX (18.2 kHz), and NLK $(24.8 \mathrm{kHz})$ transmitters monitored at Suva $\left(18.2^{\circ} \mathrm{S}, 178.4^{\circ} \mathrm{E}\right)$, Fiji. Apart from the higher class solar flares ( $C$ to X), a solar flare of class B8.5 also produced enhancements both on the amplitude and phase. The amplitude enhancements in NLK, NPM, and NWC signals as a function of peak solar flare X-ray flux in decibel (dB; relative to $1 \mu \mathrm{W} / \mathrm{m}^{2}$ ) shows that the relationship curve is steeper and quite linear between the flare power levels of 0 to $15 \mathrm{~dB}$; below $0 \mathrm{~dB}$, the curve gets less steep and flattens towards $-5 \mathrm{~dB}$ flare power level, while it also gets less steep above $15 \mathrm{~dB}$ and almost flattens above $20 \mathrm{~dB}$. In general, the level of amplitude enhancement for NLK signal is higher than that for NPM and NWC signals for all solar flares. The enhancement in the amplitude and phase of VLF signals by solar flares is due to the increase in the D-region electron density by the solar flare-produced extra ionization. The modeling of VLF perturbations produced by B8.5 and C1.5 classes of solar flares on 29 January 2007 using LWPC (Long Wave Propagation Capability) V2.1 codes show that reflection height $(H)$ was reduced by 0.6 and $1.2 \mathrm{~km}$ and the exponential sharpness factor $(\beta)$ was raised by 0.010 and $0.005 \mathrm{~km}^{-1}$, respectively. Out of seven storms with Dst $<-50 \mathrm{nT}$, only the intense storm of 14 to 16 December 2006 with a minimum Dst of $-145 \mathrm{nT}$ has shown a clear reduction in the signal strength of NWC and NPM sub-ionospheric signals due to storm-induced reduction in the D-region electron density.
\end{abstract}

Keywords: VLF perturbations; Solar flares; Geomagnetic storms; D-region ionosphere

\section{Background}

The solar flares and geomagnetic storms are the phenomena associated with the space weather. The solar flares, particularly with X-ray having wavelengths typically of tenths of a nanometer, penetrate the D-region of the ionosphere and increase the electron density via extra ionization (e.g. Mitra 1974). The increase in the D-region electron density can produce significant perturbations in the received phase and amplitude of VLF signals propagating in the Earthionosphere waveguide (EIWG). The normal unperturbed daytime D-region from which VLF signals are usually reflected is maintained mainly by direct Lyman- $\alpha$ radiation $(121.6 \mathrm{~nm})$ from the sun that partially ionizes the minor neutral constituent nitric oxide (at a height around $70 \mathrm{~km}$ ). Under normal conditions, the solar X-ray flux is too small

\footnotetext{
* Correspondence: kumar_ab@usp.ac.fj

School of Engineering and Physics, The University of the South Pacific, Suva, Fiji
}

to be a significant source for ionizing the D-region; however, when a solar flare occurs, the X-ray flux from the sun increases dramatically. The X-ray flux with wavelengths appreciably below $1 \mathrm{~nm}$ penetrates down to the D-region and markedly increases the ionization rate of the neutral constituents particularly nitrogen and oxygen hence increases the D-region electron density.

The X-ray emissions from solar flares do not penetrate down to the Earth's surface so the ground-based detectors could not determine their intensities. Solar flare data are nowadays widely used by researchers to study the changes in the amplitude and phase of VLF transmissions in relation to the increase in solar X-ray flux. McRae and Thomson (2004) using solar flare-induced perturbations in the received VLF phases and amplitudes of Omega, Japan, NPM, Hawaii, and NLK, USA, signals at Dunedin, New Zealand, have studied the changes in ionospheric parameters, $H^{\prime}$ and $\beta$ as a function of solar

\section{穴 Springer}


$\mathrm{X}$-ray flux. $H^{\prime}$ is a measure of the reflection height in $\mathrm{km}$ and $\beta$ is a measure of the sharpness or rate of change of electron density with height at the lower edge of the Dregion. Using the Long Wave Propagation Capability (LWPC) V2.1 and ModeFinder computer codes, McRae and Thomson (2004) found that the increase in the ionization due to the flares lowers the $H^{\prime}$ almost in proportion to the logarithm of the X-ray flux intensity from a typical mid-day unperturbed value of about $71 \mathrm{~km}$ down to about $58 \mathrm{~km}$ for a solar flare of X5 class and increases $\beta$ from $0.39 \mathrm{~km}^{-1}$ up to a saturation level of about $0.52 \mathrm{~km}^{-1}$. Thomson et al. (2004, 2005), for solar flares of $\mathrm{X}$ class found that the phase perturbations on NLK, NDK (USA), NPM and Omega (Australia) signals received at Dunedin show greater proportionality (correlation) with the X-ray flux over the amplitude. The GOES detectors saturate for solar flares above X17 classes. By extrapolating the phase profiles during the strong solar flares, Thomson et al. (2005) estimated the peak value of the X-ray flux that corresponded to solar flare of X45 class. Grubor et al. (2005) from measurement of GQD, Anthon ( $54^{\circ} 53^{\prime} \mathrm{N}, 17^{\circ} \mathrm{W}, 22.1 \mathrm{kHz}$ ) transmitter signal at Belgrade $\left(44^{\circ} 51^{\prime} \mathrm{N}, 20^{\circ} 23^{\prime} \mathrm{E}\right)$ for the solar flare events that occurred during 2004 and 2005, a low solar activity period, found that flares with classes $C$ to $\mathrm{X}$ can perturb the amplitude of VLF signals propagating in the EIWG. Recently, Raulin et al. (2013) examined the effects of Lyman- $\alpha$ excess during solar flares on the D-region ionosphere using the VLF phase measurements. They found that while the phase changes using the VLF technique may be a good indicator of quiescent Lyman- $\alpha$ variation along the solar cycle, they cannot be used to scale explosive Lyman- $\alpha$ emission during flares.

The second phenomenon associated with space weather effects is geomagnetic storms, which is a temporary disturbance in the Earth's magnetosphere caused by a solar wind shock wave and/or cloud of magnetic field which interacts with the Earth's magnetic field. It is also well known that like solar flares, magnetic storms are accompanied by ionospheric disturbances which can occur up to the D and E regions (e.g., Antonova et al. 1996). Communication and navigation systems such as VLF, LF, and GPS can be significantly affected by ionospheric perturbations associated with the auroral electrojet and high-energy particle precipitation during high geomagnetic activity and can extend to the mid-latitudes (Inan et al. 1985). However, much of the focus of the impact of geomagnetic storms on the ionosphere has been at high latitudes (e.g., Kikuchi and Evans 1983). Using the phase data of NWC signal received at Uji, Japan, a lowmid latitude path, Araki (1974) found that the phase decreased in the nighttime in association with the main phase of two large geomagnetic storms during 1968 to 1969. Kleimenova et al. (2004) analyzed the NWC signal received at Kamchatka, a low-mid latitude path, and found distinct anomalies in the amplitude and phase. They obtained negative phase and amplitude variations of the VLF signal both during day and nighttimes during the main storm phase days, but the nighttime variations were more pronounced. There have not been other studies on magnetic storm associated subionospheric changes purely at low latitude paths; however, there have been several studies on VLF perturbations due to storm-induced energetic electron precipitation at mid and high latitudes (e.g., Peter et al. 2006; Clilverd et al. 2010).

In this paper, we examine the effects of solar flares and geomagnetic storms (purely low latitude) on VLF paths during the period December 2006 to 2008, a period of unprecedented minimum during solar cycles 23 and 24 which has provided us with a unique opportunity to study the low latitude D-region response to space weather effects. During this extended period of solar minimum, the ionosphere was contracted to the lowest level (Heelis et al. 2009; Liu et al. 2011). The signal perturbations due to solar flares of varying intensities and any relationship between them are investigated. Typical VLF perturbation events associated with solar flares are modeled using LWPC to determine the D-region parameters. The NWC and NPM signals have been analyzed for any variation in their average signal strengths during complete daylight and nighttime propagation conditions to study the geomagnetic storm effects.

\section{Methods}

We use a software-based VLF phase and amplitude logger known as 'SoftPAL' to record the amplitude and phase of VLF transmitter signals that are MSK (Minimum Shift Key) modulated (Dowden and Adams 2008; Kumar et al. 2008). The transmitter signals were recorded continuously with a time resolution of $0.1 \mathrm{~s}$ (sampling frequency $10 \mathrm{~Hz}$ ), using GPS-based timing. The 1-min average values of the amplitudes and phases obtained from 0.1-s resolution recording have been utilized to display the typical changes in the amplitudes and phases of VLF signals due to the flares and storms. We study the effects of solar flares and geomagnetic storms on NWC $\left(21.82^{\circ} \mathrm{S}, 114.17^{\circ} \mathrm{E}, 19.8 \mathrm{kHz}\right.$, $1,000 \mathrm{~kW}), \mathrm{NPM}\left(21.42^{\circ} \mathrm{N}, 158.15^{\circ} \mathrm{W}, 21.4 \mathrm{kHz}, 424 \mathrm{~kW}\right)$, $\operatorname{VTX}\left(8.39^{\circ} \mathrm{N}, 77.75^{\circ} \mathrm{E}, 18.2 \mathrm{kHz}\right.$, power not known), and NLK $\left(48.20^{\circ} \mathrm{N}, 121.92^{\circ} \mathrm{W}, 24.8 \mathrm{kHz}, 192 \mathrm{~kW}\right)$ transmitter signals received at Suva whose TRGCPs are shown in Figure 1. The plots of the amplitude and phase perturbations on the above signals are illustrated for typical solar flare events, while the interesting feature of relationship between amplitude perturbation and solar flare power is examined and presented for the NLK, NPM, and NWC signals when their TRGCPs were in daylight for the solar flare events during December 2006 to 2008. 


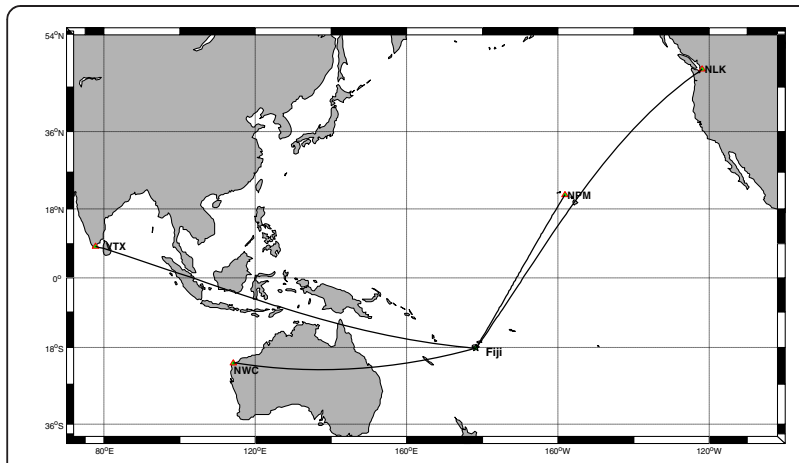

Figure 1 Map showing the transmitter and receiver sites and their TRGCPs.

The solar X-ray imager abroad GOES satellite records the X-ray fluxes in two wavelength bands: (1) 0.1 to $0.8 \mathrm{~nm}$, referred to as 'long' or 'XL', and (2) 0.05 to $0.4 \mathrm{~nm}$, referred to as 'short' or 'XS.' Solar flares are classified in A, B, C, M, and X classes according to the peak flux (in Watts per square meter, $\mathrm{W} / \mathrm{m}^{2}$ ) in the ' $\mathrm{XL}$ ' band. The flux intensities corresponding to different classes of the flares are given in Table 1. In this work, the solar flux data in the 'XL' band are used. The period of data analysis 2006 to 2008 (average sunspot number $R_{z}=12$ ) is of importance in the sense that it falls under a period of solar minimum and especially under extended solar minimum of solar cycle 23/24. The solar activity in 2007 to 2009 was extremely prolonged low, for which the end of the solar minimum of solar cycle 23 came later at least 2 years than was expected (Liu et al. 2011). Fewer solar flares occur during the low solar activity period, but the effect of even weak class solar flares can be seen on VLF. The 1-min average values of both solar flux and amplitude and/or phase of VLF signals are considered for analysis.

A count was made for the number of solar flares occurring during this period, which is given in Table 2 . Only C, M, and X class flares were counted as these mostly produced clear detectable effects on the amplitudes and phases of VLF transmissions. It can be clearly seen that $\mathrm{C}$ class flares occurred most often, $M$ class less, and $\mathrm{X}$ class least. The monthly occurrence of flares was highest during December 2006. The X class flares

Table $1 \mathrm{X}$-ray $(0.1$ to $0.8 \mathrm{~nm}$ ) peak flux intensities for different classes of flares

\begin{tabular}{lc}
\hline Class & Peak flux intensities $\left(\mathrm{W} / \mathrm{m}^{2}\right)$ \\
\hline A & $1<10^{-7}$ \\
B & $10^{-7} \leq 1<10^{-6}$ \\
C & $10^{-6} \leq l<10^{-5}$ \\
$M$ & $10^{-5} \leq l<10^{-4}$ \\
$X$ & $10^{-4} \leq 1$ \\
\hline
\end{tabular}

Table 2 Occurrence of solar flares of different classes as observed by GOES for the periods of data analysis

\begin{tabular}{lccc}
\hline \multirow{2}{*}{ Months } & \multicolumn{3}{c}{ Number of flares } \\
\cline { 2 - 4 } & $\mathbf{C}$ & $\mathbf{M}$ & $\mathbf{X}$ \\
\hline Dec. 2006 & 53 & 5 & 4 \\
Jan. 2007 to Dec. 2007 & 72 & 10 & 0 \\
Jan. 2008 to Dec. 2008 & 13 & 2 & 0 \\
Total & 138 & 17 & 4 \\
\hline
\end{tabular}

occurred only during December 2006. Solar flareinduced perturbations in the amplitude $(\Delta A)$ of VLF transmissions were determined using the method as demonstrated in Figure 2. The value of $\triangle A$ is obtained by $\Delta A=A_{\text {peak }}-A_{\text {background, }}$, where $A_{\text {peak }}$ is the value of peak amplitude during solar flare and $A_{\text {background }}$ is the monthly mean value of amplitude at the time of peak amplitude. This method is similar to that used by Todoroki et al. (2007). The perturbation in phase is not determined as it was unstable during most of the flares. Solar flare-induced perturbations in all cases showed enhancement in the amplitude and advances in the phase under stable phase conditions. Solar flare effects were observed only when the TRGCP was entirely or partly in the daylight region.

To study the storm effects on VLF propagation, we use only the amplitude data for the NWC and NPM signals which cover purely low-latitude paths. Due to complicated modulation of the VLF transmitter signals, it is often difficult to accurately record the phase for long periods (several hours) and it does not follow the same values as before the temporary transmitter switch off; hence, continuous phase data was not available for significant periods during the storm analysis. During the period of data analysis (Dec. 2006 to 2008), seven storms

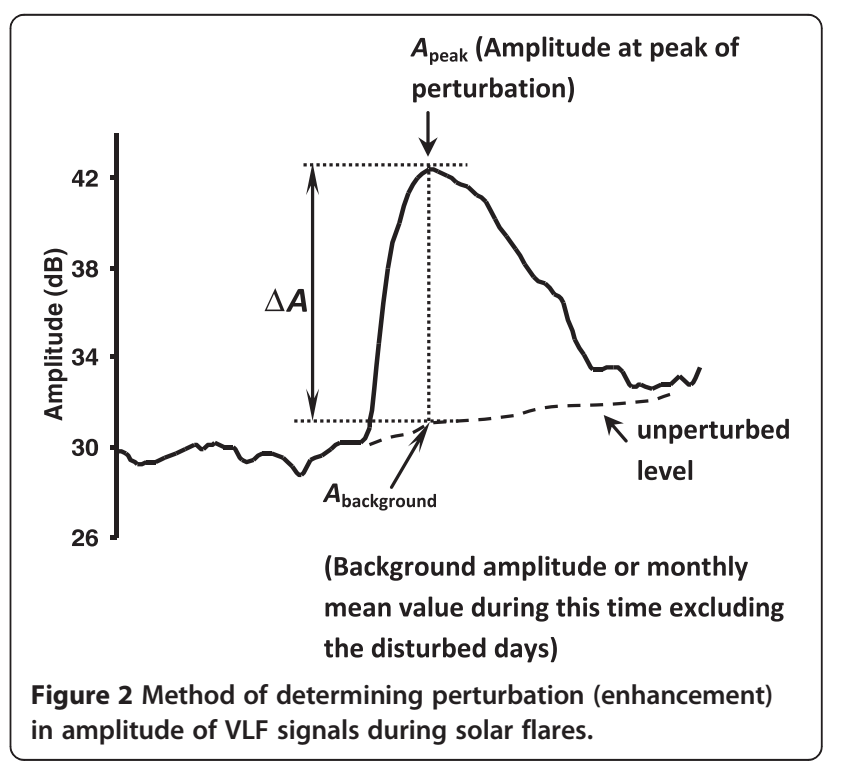


(disturbance storm time index $(D s t)<-50 \mathrm{nT})$ were registered, for which the VLF amplitude data were analyzed for a day before the storm, on the storm day, and 3 days after the storm for any perturbations. Since the amplitude variations are large during the terminator transitions (during occurrence of day and night minima), only the amplitude during the period when the TRGCP was in complete daylight and darkness and with an almost constant signal level were analyzed for any perturbations. The average values for the daytime and nighttime signal strengths for both NWC and NPM were calculated for the period 12 to 18 December 2006. This was the period during which only one storm out of the seven studied, displayed an anomalous effect on the VLF signals.

\section{Results and discussion}

\section{Solar flare associated VLF perturbations}

The solar flare-induced perturbations measured in the amplitudes and phases (where possible) on four VLF signals propagating over long sub-ionospheric paths were studied. Few such case studies on 05, 13, and 14 December 2006, and on 29 January 2007 are presented in Figures 3 and 4. The X-ray fluxes from the GOES satellites, which vary proportionally in the time and level of observed VLF perturbations (enhancements in our case), are also utilized. The solar flares of 05,13 , and 14 December were associated with solar radio bursts (SRB) which drastically reduced the carrier-to-noise ratio $(\mathrm{C} / \mathrm{No})$ of the Global Positioning System signals and the SRB associated with 05 December flare was strong enough to substantially degrade GPS tracking and positioning accuracy (Carrano et al. 2009). The VLF perturbations associated with strong lightnings also occur when the TRGCPs are in the daylight but they can be easily distinguished from solar flare associated perturbations on the basis of their low level $(0.2$ to $0.5 \mathrm{~dB})$ and short durations (10 to 30 s) (Kumar et al. 2008; Kumar and Kumar 2013).

Two solar flares of class M1.8 and X9.0 occurred on 05 December 2006 and the effects of these were observed on the amplitude of NWC signal. The M1.8 flare started at 07:45 UT (19:45 LT) and ended at 8:06 UT while the X9.0 flare started at 10:18 UT (22:18 LT) and ended at 10:45 UT. The VTX signal was off during this time while the NPM and NLK TRGCPs were in dark; hence, no effect was apparent on their signals. The variations in the amplitude of NWC signal along with the respective X-ray flux intensities during both the flares are shown in Figure 3a, b. The $\triangle A$ of 3.6 and $13.4 \mathrm{~dB}$ for the M1.8 and X9.0 flares, respectively, was estimated. A feature of interest seen here is the time difference in the peak occurrences of the solar flux intensities and the respective amplitudes during these flares. These peaks are marked with arrows in the panels (a) and (b). A time delay $(\Delta t)$ of about $4 \mathrm{~min}$ is obtained for the M1.8 flare, with the amplitude peak occurring after the solar flare flux peak. Such time delays have been reported by Grubor et al. (2005) and by Zigman et al. (2007). They attributed this time delay to the electron density peak which occurs slightly after the flux peak and provides the information about the ionospheric response to the enhanced flare flux. According to Zigman et al. (2007), the time delay is needed for the D-region recombination-ionization processes to recover balance under the enhancement of X-ray flare. This time is also an important key to solve the continuity equation of electrons and identify the responses of ionospheric D-region to solar flares. The X9.0 and X3.4 flares show a time difference of about $2 \mathrm{~min}$ between the flux and amplitude peaks with the amplitude peak occurring before the flare flux peak in contrast to what is expected. These are the flares for which the X-ray flux initially increased sharply and then gradually in contrast to other flares when increase in flux was sharp throughout. It seems that the amplitude did not respond proportionally to the gradual increase in the flux; however, the initial response of amplitude was always slightly after the increase in X-ray flux intensity as in all the events shown. The plausible explanation for such negative time lags could be that during the gradual increase in flux during the solar flares, the recombination/attachment processes do not allow the D-region electron density to increase further; hence, the amplitude does not continue to increase. In addition, because the X9.0 flare occurred during the time of the sunset minima on NWC, the amplitude did not respond proportionately to the solar flux intensity. The effect of flares that occur during sunset/sunrise transition are not very apparent because the day/light changes of propagation conditions dominate over the effect of solar flares even for the strongest flares (Grubor et al. 2005). Similar time delays when the amplitude peak occurs earlier than the flare flux peak have been reported for some events by authors like Tan et al. (2013) who used the NWC signal recorded at Tay Nguyen University, Vietnam. However, the mechanism of this phenomenon, which is very rare, has not been clearly understood.

Another flare of class X3.4 started at 2:14 UT (14:14 LT) and ended at 2:57 UT on 13 December 2006 with the peak flux at 2:40 UT. During this time, the NPM and NLK TRGCPs were in daylight while NWC and VTX transmitters were off. The variations in the amplitudes of NPM and NLK signals during solar flare along with the X-ray flux intensity are presented in Figure 3c, $\mathrm{d}$, respectively. The $\Delta A$ values found were $5.8 \mathrm{~dB}$ for NPM and $9.9 \mathrm{~dB}$ for NLK signal. The phase perturbations could not be obtained, as phase was unstable for both the signals.

A solar flare of class X1.5 occurred on 14 December 2006 at 21:07 UT (09:07 LT) and ended at 22:26 UT 


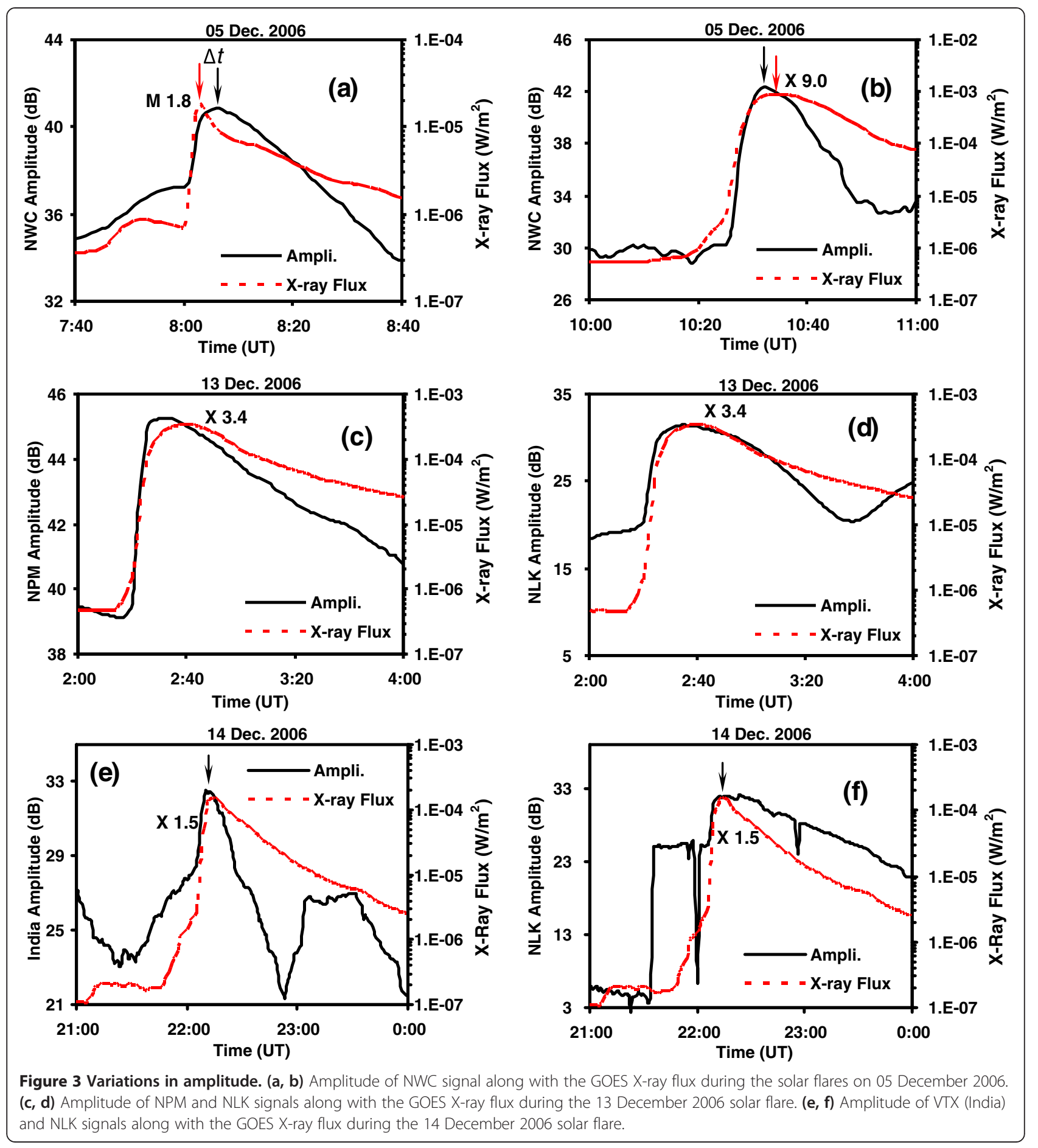

with its peak flux at 22:15 UT. The amplitudes of VTX and NLK with the corresponding solar flux intensity are presented in Figure 3e, $\mathrm{f}$. The values of $\Delta A$ were found to be 4 and $8.8 \mathrm{~dB}$ for VTX and NLK signals, respectively. Though not very apparent, but still the peak of the amplitude of the signal seems to correspond well with the peak of the X-ray flux. For NLK signal, the amplitude rises proportionally with flux up to the peak value but decreases slowly when compared to the flux after the peak. The low amplitude value uptil 21:30 UT represents the signal being off air while the short negative spikes are due to power offs in the VLF telemetry.

Two solar flare events occurred on 29 January 2007: (1) class B8.5 that began at 21:34 UT (09:34 LT) and ended at 21:45 UT and (2) class C1.5 that began at 22:32 UT (10:32 LT) and ended at 23:21 UT. The variation in 

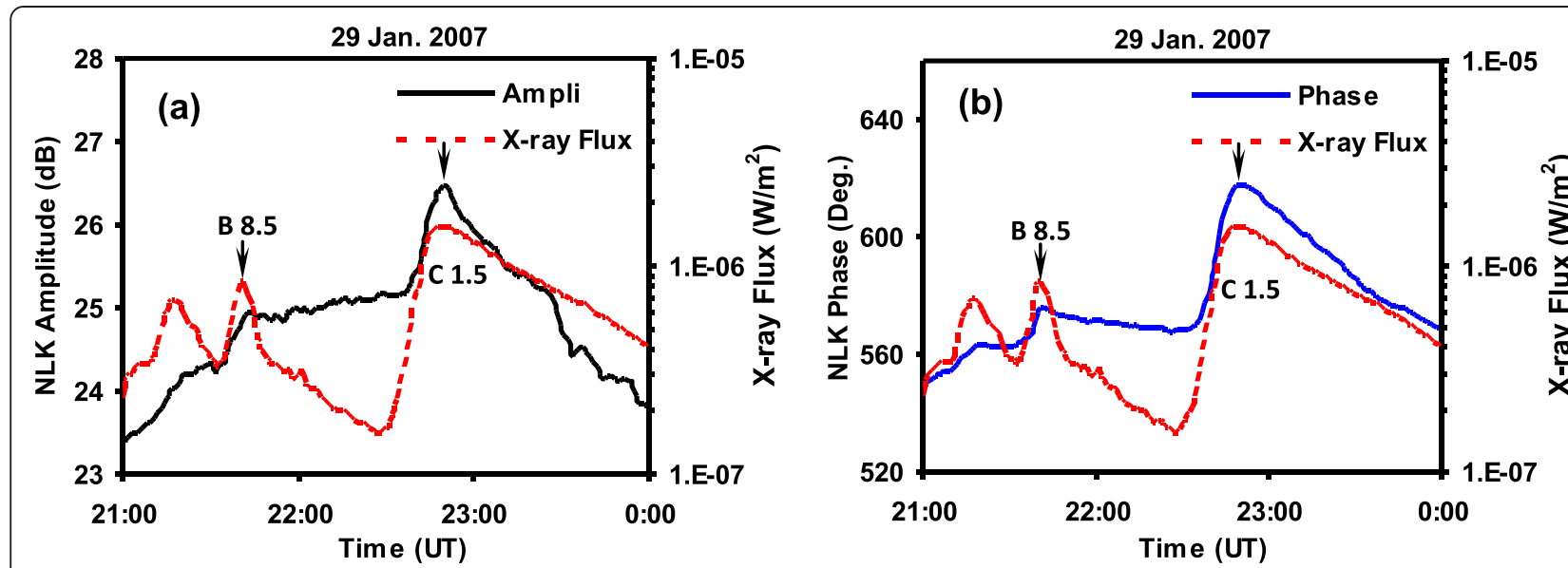

Figure 4 Variation in amplitude (a) and phase (b) of NLK signal. This is along with the GOES X-ray flux during the solar flares on 29 January 2007.

the amplitude and phase of NLK signal during the solar flares along with the X-ray flux intensity are presented in Figure 4a, b, respectively. Pacini and Raulin (2006) concluded that minimum energy input necessary to produce any sudden phase or amplitude anomaly is about $2.5 \times 10^{-6} \mathrm{~J} \mathrm{~m}^{-2}$ during solar minimum and about $7.0 \times$ $10^{-6} \mathrm{~J} \mathrm{~m}^{-2}$ during solar maximum. To our knowledge, solar flares of class $\mathrm{C}$ and above have been reported so far to show detectable effect on VLF transmissions. However, at our station on 29 January 2007, the solar flare of magnitude less than $\mathrm{C}$ class $\left(\mathrm{B} 8.5,8.5 \times 10^{-7} \mathrm{~J} \mathrm{~m}^{-2}\right)$ showed detectable enhancement in the amplitude and phase of NLK signal. The values of $\Delta A$ were 0.4 and $1.0 \mathrm{~dB}$ for the $\mathrm{B} 8.5$ and $\mathrm{C} 1.8$ classes, respectively, and the phase perturbations $(\Delta \phi)$ were $8^{\circ}$ and $40^{\circ}$ due to these two flares, respectively. The NPM signal was off at that time. No effect is seen on the NWC and VTX signals which may be due to transition of the terminator from the receiver to the transmitter. From the results presented here for the 29 January 2007 solar flares, we can see that both the phase and amplitude vary in proportion with the logarithm of the X-ray flux. The phase and amplitude could be useful and convenient for extrapolating the $\mathrm{X}$-ray flux when the GOES detectors saturate during very strong solar flares to determine the actual flux densities associated with the strong solar flares. GOES detectors saturate for solar flares stronger than X17 class (Thomson et al. 2005).

Out of 152 flares listed in Table 2, 42 solar flares occurred when the NLK/NPM TRGCPs were in daylight but the effect was seen only for 25 on NLK and 22 on NPM. Forty-five flares occurred when the NWC path was in daylight but effect on NWC signal was seen only for 12 flares. The VLF perturbations were not noticed for all the flares occurring during the daylight either because the transmitters were off air (no data available) or simply the effect was not visible. The amplitude perturbations, $\triangle A$, in $\mathrm{dB}$ at the time of peak of the 25 solar flares on NLK, 22 on NPM, and 12 on NWC during December 2006 to 2008, that have shown effect on the signals, are plotted as the function of X-ray solar flare power in $\mathrm{dB}$ over $1 \mu \mathrm{W} / \mathrm{m}^{2}$ in Figure 5 . The flares ranged from B8.5 to X6.5 classes. All the flares studied here occurred when the TRGCPs were in the daylight. The best-fit curves for the amplitude perturbation versus the flare power for the three signals resemble the form previously obtained by McRae and Thomson (2004) and Thomson et al. (2005). It can be noted that the curve is steeper and quite linear between the flare power levels of 0 to $15 \mathrm{~dB}$. Below $0 \mathrm{~dB}$, the curve gets less steep and flattens towards $-5 \mathrm{~dB}$ flare power level. It also gets less steep above $15 \mathrm{~dB}$ and almost flattens as previously reported by McRae and Thomson (2004). The peak phase perturbation could not be plotted with the respective peak flare power because the phase was not stable for most of the time; hence, enough data points could not be obtained. The variation between the VLF amplitude perturbation level and the flux intensity during solar flares for the three paths, also shows that the level of perturbation is highest for the NLK path (maximum of approximately $11.5 \mathrm{~dB}$ ), less for NPM (approximately $6.0 \mathrm{~dB}$ ) and least for the NWC path (approximately 3.5 dB). McRae and Thomson (2004) obtained similar results for NLK and NPM to Dunedin paths where NLK displayed perturbations of up to $8 \mathrm{~dB}$ while NPM showed perturbations of up to $4 \mathrm{~dB}$ for same flares. They stated that at higher VLF frequencies (20 to $30 \mathrm{kHz})$, the flare enhances the amplitude perturbations. The amplitude enhancements in $\mathrm{dB}$ can be seen to be roughly proportional to the flare $\mathrm{X}$-ray flux in $\mathrm{dB}$ for increasing frequencies. The increasing complexity of the amplitude enhancements as a function of frequency at the highest 


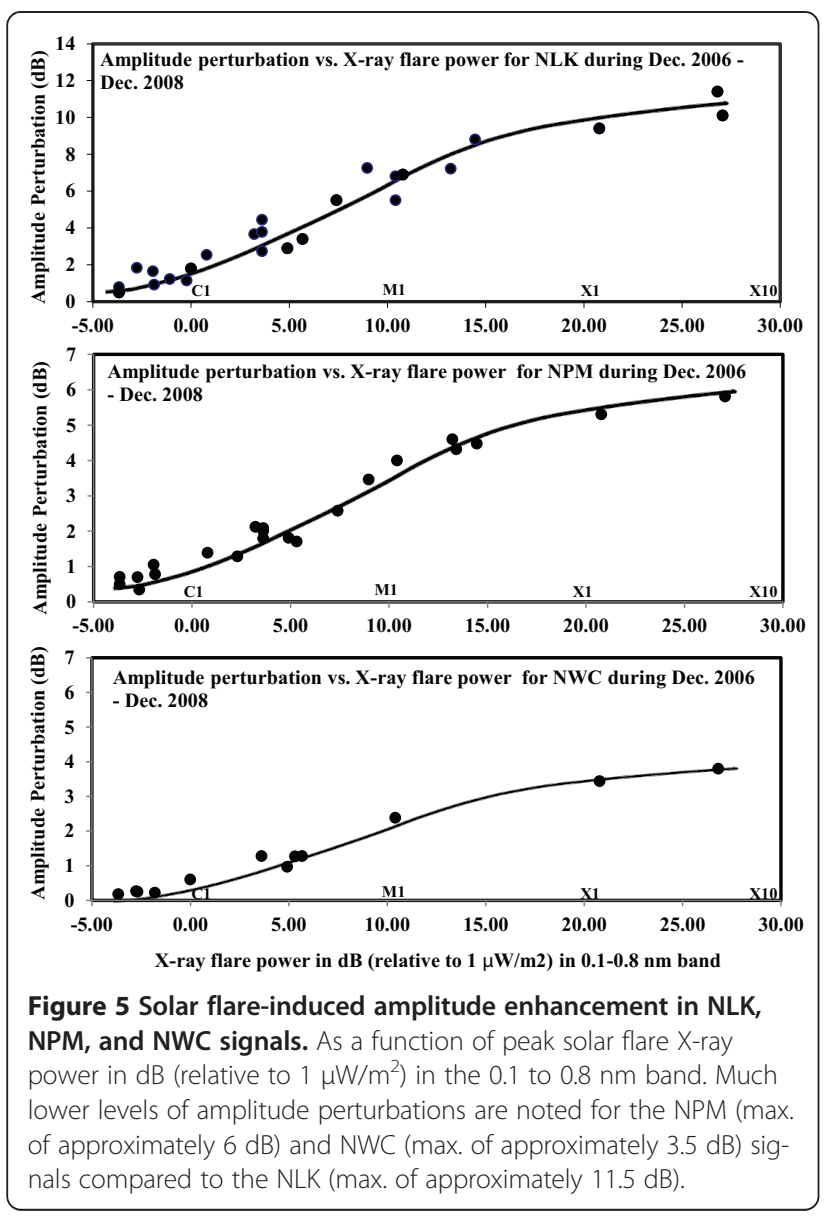

VLF frequencies is due to the amplitude of the secondorder TM mode becoming comparable with that of the first-order TM mode at the receiver. The difference in the level of amplitude perturbations could also be different for different paths like that of NWC and NLK/NPM for same flares because the directions in which the flares are directed. One of the paths could be under more daylight when compared to the other (different solar zenith angles); hence, it may be more susceptible to flareinduced perturbation.

The two solar flare perturbation events of 29 January 2007 (both the amplitude and phase available) have been modeled to determine the D-region electron density changes associated with these flares. The observed VLF amplitude and phase perturbations on NLK signal are simulated using the LWPC V2.1 code for Wait's model of the lower ionosphere characterized by two parameters: the sharpness $\left(\beta\right.$ in $\left.\mathrm{km}^{-1}\right)$ and reflection height $\left(H^{\prime}\right.$ in $\left.\mathrm{km}\right)$. The solar zenith angle values for mid TRGCP of NLK-Suva path at the times of occurrence of the flares were calculated which were then used to estimate the values of $H^{\prime}$ and $\beta$ using the relationship given by McRae and Thomson (2000).
By varying the values of $H^{\prime}$ and $\beta$ so as to match the observed perturbed amplitude $(A+\Delta A)$ and phase $(\phi+$ $\Delta \phi)$, the values of $H^{\prime}$ and $\beta$ for solar flare-perturbed ionosphere were obtained. The values of $H^{\prime}$ and $\beta$ for the unperturbed daytime ionosphere were found to be $H^{\prime}=72.6 \mathrm{~km}$ and $\beta=0.350 \mathrm{~km}^{-1}$ during the $B 8.5$ flare, and $H^{\prime}=75.0 \mathrm{~km}$ and $\beta=0.300 \mathrm{~km}^{-1}$ during the $C 1.5$ class flare. By adjusting the values of $H^{\prime}$ (in intervals of $0.05 \mathrm{~km}$ ) and $\beta$ (in intervals of $0.001 \mathrm{~km}^{-1}$ ) to match the values of perturbed amplitude and phase during the two flares, the estimated values came as $H^{\prime}=72.0 \mathrm{~km}$ and $\beta=0.360 \mathrm{~km}^{-1}$ for the B8.5 class flare, and $H^{\prime}=73.8 \mathrm{~km}$ and $\beta=0.305 \mathrm{~km}^{-1}$ for the $C 1.5$ class flare. In both the cases, the $H^{\prime}$ decreased $\left(\Delta H^{\prime}=0.60 \pm 0.05 \mathrm{~km}\right.$ for B8.5 and $\Delta H^{\prime}=1.20 \pm 0.05 \mathrm{~km}$ for C1.5 flare) while the $\beta$ increased $\left(\Delta \beta=0.010 \pm 0.001 \mathrm{~km}^{-1}\right.$ for $\mathrm{B} 8.5$ and $\Delta \beta=$ $0.005 \pm 0.001 \mathrm{~km}^{-1}$ for $\mathrm{C} 1.5$ flare). The results show that there is significant change in the $H^{\prime}(0.6$ and $1.0 \mathrm{~km})$ for $\mathrm{B}$ and $\mathrm{C}$ class flares respectively; however, there is no significant change in $\beta\left(0.01\right.$ and $\left.0.005 \mathrm{~km}^{-1}\right)$ for both the flares which is within the model accuracy range. The $\mathrm{X}$-rays from solar flares ionize the neutral atmosphere at D-region heights (40 to $90 \mathrm{~km}$ ) greatly increasing the electron densities there and thus markedly lowering the effective VLF reflection height. This lowering of the reflection height is the main cause of the VLF amplitude and phase increases observed during the flares. In addition, the electron density profile at the lower edge of the D-region also sharpens during flares in the sense that the rate of increase of electron density with height $(\beta)$ increases, and this also affects the VLF amplitude and phase at the receiver (Thomson and Clilverd 2001; McRae and Thomson 2004). The relationship between the flare power and the changes in the $H^{\prime}$ and $\beta$ during solar flares of varying intensities has been presented by Thomson et al. (2005). An almost linear reduction of $H^{\prime}$ with (the logarithm of) the flare flux was obtained for $\mathrm{C} 1$ to $\mathrm{X} 45$ class flares. The $\beta$ was found to increase linearly with the flare flux for $\mathrm{C} 1$ to $\mathrm{X} 1$ classes, levels off for $\mathrm{X} 1$ to $\mathrm{X} 5$, and started increasing linearly again after $\mathrm{X} 10$ class to very large flares (up to X45).

\section{Geomagnetic storm associated VLF perturbations}

The geomagnetic storms occur rarely during the solar minimum. However, few moderate and one intense storm occurred during December 2006 to 2008. We have used Dst values to classify storms of various levels of strength as follows: moderate, $-100 \mathrm{nT} \leq D s t<-50 \mathrm{nT}$; intense, -200 $\mathrm{nT} \leq D s t<-100 \mathrm{nT}$; and very intense, $D s t<-200 \mathrm{nT}$. The Dst index is a measure of ring currents and has been the most widely used geomagnetic index to classify the storms at low latitudes which affect the horizontal component of the terrestrial magnetic field. The ring current is formed primarily through injection and subsequent trapping of 
Table 3 Details of intense geomagnetic storms with Dst $<-50$ $\mathrm{nT}$ and effects on VLF signals during data analysis

\begin{tabular}{lcc}
\hline Storm days & Dst & VLF amplitude perturbations \\
\hline 14 to 16 Dec 2006 & $-146 \mathrm{nT}$ & Seen on NWC and NPM \\
24 to 25 March 2007 & $-72 \mathrm{nT}$ & None \\
21 to 22 May 2007 & $-63 \mathrm{nT}$ & None \\
25 to 26 October 2007 & $-52 \mathrm{nT}$ & None \\
20 to 21 November 2007 & $-59 \mathrm{nT}$ & No data \\
8 to 9 March 2008 & $-72 \mathrm{nT}$ & None \\
11 to 12 October 2008 & $-54 \mathrm{nT}$ & None \\
\hline
\end{tabular}

energetic particles into the radiation belts of the inner magnetosphere (Daglis and Thorne 1999). Out of the seven geomagnetic storms (six moderate and one intense) under study during the data analysis period, only an intense storm of 14 to 16 December 2006 with its main phase onset on 14 December at about 22 UT and lasting up to 06 UT on 15 December 2006, has shown convincing evidence of VLF perturbations on NWC and NPM signals as indicated in Table 3. This storm was most intense compared with others on the list with $D s t=-146 \mathrm{nT}$ and was associated with coronal mass ejection and a solar flare of class X1.5. The diurnal plots for both NWC and NPM signals were closely inspected for the period 12 to 18 December 2006. No apparent increase in signal fluctuations were noticed during day or nighttime as reported by previous researchers such as Peter et al. (2006) who reported amplitude fluctuations in the nighttime data and Belrose and Thomas (1968) who reported fluctuations in the phase during twilight and nighttime during the geomagnetic storms in the mid-latitude regions. The relevant phase data which were intercepted by short-term switching off of transmitters have not been used for analysis, since phase does not follow the same value as before the transmitter was switched off. However, an interesting feature of average signal strength variation was identified from the data analysis. The average signal strengths during day and nighttimes for both the signals were obtained from the amplitude data and are presented in Figure 6. The period 00 to 04 UT and 12 to 16 UT resembled purely daytime and nighttime paths for the NWC signal, respectively, while 22 to 02 UT and 08 to 16 UT represented day and nighttime respectively for the NPM signal. The average signal strength for the NWC signal was reduced by approximately $3.2 \mathrm{~dB}$ during the daytime and $2.5 \mathrm{~dB}$ during the nighttime on 15 December 2006 in association with the main storm phase. Likewise, the average signal strength of NPM signal showed a reduction of about $1.0 \mathrm{~dB}$ during daytime and $2.5 \mathrm{~dB}$ during the nighttime on the same day. A similar reduction in the signal amplitudes of VLF transmissions were reported by previous researchers during the main phase of the storms they studied (e.g., Kleimenova et al. 2004; Peter et al. 2006). It can be noted from Figure 6 that the average signal strengths started showing dip/decrease on 15 December at 00:30 UT (obtained from the diurnal plots) which corresponds to the storm main phase and had a lasting effect until 16 December at 23:00 UT during the recovery phase of the storm thus showing an effect of this storm on the D-region for about $46 \mathrm{~h}$. The most likely reason for this longer term decrease in the amplitude commencing during the main phase of the storm is due to the slow recombination of ions and free electrons and slower molecular diffusion at D-region altitudes as compared to those at the $\mathrm{F}$ region altitudes during the storms (Richmond and Lu 2000). Perturbations in the composition, temperature, and wind at one altitude are rapidly transmitted by diffusion which is lower at low altitudes, e.g., diffusive time are about 1 day at $125 \mathrm{~km}$, and $1 \mathrm{~h}$ at $240 \mathrm{~km}$ (Richmond and Lu 2000). Therefore, storm-induced compositional (electron density) changes in the D-region last longer and produce the long duration changes in subionospheric propagation. Further investigations on the D-region parameter changes using the LWPC code and most likely mechanisms for such long-term perturbations are underway which may appear as separate study in future.

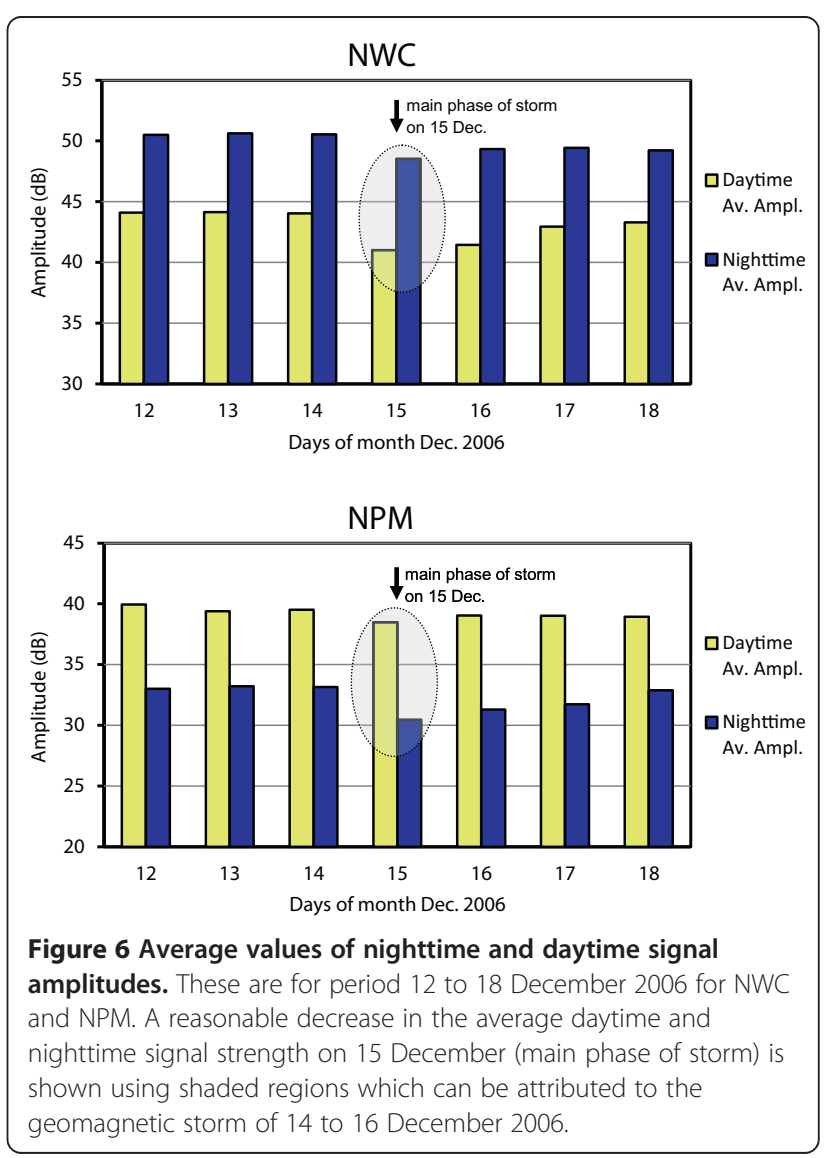




\section{Conclusions}

The VLF amplitude and phase (wherever available) data have been used to study the effects of space weather phenomena; solar flares and geomagnetic storms (purely low latitude) on the D-region ionosphere during solar minimum. The amplitude and phase of VLF signals exhibit perturbations with increased solar activity during flares which can last for several minutes. Likewise, VLF perturbations have been observed only under intense geomagnetic storm of 2006 resulting in signal level decrease lasting for about 2 days. Based on the data analysis for the period December 2006 to 2008, we summarize our findings as follows:

1. During solar flares, both the VLF amplitude and phase are found to vary with the logarithm of the $\mathrm{X}$-ray flux. From our data, VLF amplitude being very stable and available for very long periods when compared to the phase can be effectively used to study the relationship between the solar X-ray flux intensity and the amplitude perturbations. A best fit curve of amplitude perturbation $(\mathrm{dB})$ versus the flare power for NLK, NPM, and NWC signals reveals the following features; the curve is steeper and quite linear between the flare power levels of 0 to $15 \mathrm{~dB}$; below $0 \mathrm{~dB}$, the curve gets less steep and flattens towards $-5 \mathrm{~dB}$-flare power level, while it also gets less steep above $15 \mathrm{~dB}$ and almost flattens at around $20 \mathrm{~dB}$.

2. VLF amplitude perturbation level versus the flux intensity for solar flares on the NLK, NPM, and NWC paths shows that the level of perturbation is greatest for the NLK path (max. of approximately $11.5 \mathrm{~dB}$ ), less for NPM (max. of approximately $6.0 \mathrm{~dB}$ ) and least for the NWC path (max. of approximately $3.5 \mathrm{~dB}$ ).

3. The X-rays from solar flares have the tendency to ionize the neutral atmosphere at D-region heights which greatly increases the electron densities thus markedly lowers the effective VLF reflection height. The effects of solar flares can be observed on VLF signals for even small flares up to B class (B8.5 as reported here) and causes changes in the ionospheric D-region parameters, $H^{\prime}$ and $\beta$ during solar minimum. The $H^{\prime}$ is found to decrease while the $\beta$ is found to increase during flares and these changes are dependent on the strength/class of the flares. Generally, the greater the flare, the larger the reduction in the $H^{\prime}$ and larger the increase in $\beta$.

4. Moderate geomagnetic storms (six storms, -50 $\mathrm{nT} \geq$ Dst $>-100 \mathrm{nT}$ ) have not shown any effect on the D-region ionosphere at the low latitude as evident from no change in the strength of NWC and NPM signals recorded at a low latitude station, Suva. The observed amplitude of NPM and NWC signals clearly show depression in the average strengths both during the day and nighttimes of paths associated with intense geomagnetic storm of 14 to 16 December 2006 (Dst $=-146 \mathrm{nT})$. However, this is clearly an area of further research for low latitude paths.

\section{Competing interests}

The authors declare that they have no competing interests.

\section{Authors' contributions}

AK carried out the data analysis for solar flare and geomagnetic storm associated effects on the VLF propagation. The manuscript was prepared jointly by AK and SK. Subsequent revisions were done jointly. Both authors read and approved the final manuscript.

\section{Acknowledgement}

Authors are thankful to the Faculty Research Committee of The University of the South Pacific for research grant, which enabled them to carry out this research.

Received: 13 March 2014 Accepted: 15 July 2014

Published: 24 July 2014

\section{References}

Antonova LA, Ivanov-Kholodny GS, Chertoprud VE (1996) Aeronomy of the E-layer. Yanus, Moscow

Araki T (1974) Anomalous phase changes of transequatorial VLF radio waves during geomagnetic storms. J Geophys Res 79:4811-4816

Belrose JS, Thomas L (1968) lonization changes in the middle latitude D-region associated with geomagnetic storms. J Atmos Sol-Terr Phys 30:1397-1413

Carrano CS, Bridgwood CT, Groves KM (2009) Impacts of the December 2006 solar radio bursts on the performance of GPS. Radio Sci 44:RS0A25. doi:10.1029/2008RS004071

Clilverd MA, Rodger CJ, Gamble RJ, Ulich T, Raita T, Seppälä A, Green JC, Thomson NR, Sauvaud JA, Parrot M (2010) Ground-based estimates of outer radiation belt energetic electron precipitation fluxes into the atmosphere. J Geophys Res 115:A12304. doi:10.1029/2010JA015638

Daglis IA, Thorne RM (1999) The terrestrial ring current: origin formation and decay. Rev Geophys 37:407-438

Dowden RL, Adams CDD (2008) SoftPAL, Proceedings of the 3rd VERSIM Workshop, 15-20 September 2008. Tihany, Hungary, pp 15-20

Grubor D, Sulic D, Zigman V (2005) Influence of solar X-ray flares on the Earth-ionosphere waveguide. Serbian Astronomical J 171:29-35

Heelis RA, Coley WR, Burrell AG, Hairston MR, Earle GD, Perdue MD, Power RA, Harmon LL, Holt BJ, Lippincott CR (2009) Behavior of O+/H+ transition height during extreme solar minimum of 2008. Geophys Res Lett (USA) 36: L00C03. doi:10.1019/2009GL038652

Inan US, Carpenter DL, Helliwell RA, Katsufrakis JP (1985) Subionospheric VLF/LF phase perturbations produced by lightning whistler induced particle precipitation. J Geophys Res 90:7457

Kikuchi T, Evans DS (1983) Quantitative study of substorm-associated VLF phase anomalies and precipitating energetic electrons on November 13, 1979. J Geophys Res 88:871

Kleimenova NG, Kozyreva OV, Rozhnoy AA, Solov' eva MS (2004) Variations in the VLF signal parameters on the Australia-Kamchatka radio path during magnetic storms. Geomagn Aeron 44:385-393

Kumar S, Kumar A (2013) Lightning-associated VLF perturbations observed at low latitude: occurrence and scattering characteristics. Earth Planets Space 65 (1):25-37. doi:10.5047/eps.2012.05.019

Kumar S, Kumar A, Rodger CJ (2008) Subionospheric early VLF perturbations observed at Suva: VLF detection of red sprites in the day. J Geophys Res 113:A03311. doi:10.1029/2007JA012734

Liu L, Chen Y, Le H, Kurkin VI, Polekh NM, Lee CC (2011) The ionosphere under extremely prolonged low solar activity. J Geophys Res 116:A04320. doi:10.1029/2010JA016296

McRae WM, Thomson NR (2004) Solar flare induced ionospheric D-region enhancements from VLF phase and amplitude observations. J Atmos Sol-Terr Phys 66:77-87 
McRae WM, Thomson NR (2000) VLF phase and amplitude: daytime ionospheric parameters. J Atmos Sol-Terr Phys 62(7):609-618

Mitra AP (1974) lonospheric effects of solar flares. D. Reidel Publishing Company, Dordrecht, Holland

Pacini AA, Raulin J-P (2006) Solar X-ray flares and ionospheric sudden phase anomalies relationship: a solar cycle phase dependence. J Geophys Res 111:A09301. doi:10.1029/2006JA011613

Peter WB, Chevalier MW, Inan US (2006) Perturbations of midlatitude subionospheric VLF signals associated with lower ionospheric disturbances during major geomagnetic storms. J Geophys Res 111:AO3301

Raulin J-P, Trottet G, Kretzschmar M, Macotela EL, Pacini A, Bertoni FCP, Dammasch IE (2013) Response of the low ionosphere to X-ray and Lyman-a solar flare emissions. J Geophys Res 118: doi:10.1029/2012JA017916

Richmond AD, Lu G (2000) Upper-atmospheric effects of magnetic storms: a brief tutorial. J Atmos Sol-Terr Phys 62(20.00):1115-1127

Tan LM, Thu NN, Ha TQ (2013) Observation of the effects of solar flares on the NWC signal using the new VLF receiver at Tay Nguyen University. Sun Geosphere 8(1):27-31

Thomson NR, Clilverd MA (2001) Solar flare induced ionospheric D-region enhancements from VLF amplitude observations. J Atmos Sol-Terr Phys 63:1729-1737

Thomson NR, Rodger CJ, Clilverd MA (2005) Large solar flares and their ionospheric D region enhancements. J Geophys Res 110:A06306

Thomson NR, Rodger CJ, Dowden RL (2004) Ionosphere gives size of greatest solar flares. Geophys Res Lett 31:L06803

Todoroki Y, Maekawa S, Yamauchi T, Horie T, Hayakawa M (2007) Solar flare induced $D$ region perturbation in the ionosphere, as revealed from a short-distance VLF propagation path. Geophys Res Lett 34:L03103

Zigman V, Grubor D, Sulic D (2007) D-region electron density evaluated from VLF amplitude time delay during X-ray solar flares. J Atmos Sol-Terr Phys 69:775-792

doi:10.1186/1880-5981-66-76

Cite this article as: Kumar and Kumar: Space weather effects on the low latitude D-region ionosphere during solar minimum. Earth, Planets and Space 2014 66:76

\section{Submit your manuscript to a SpringerOpen ${ }^{\circ}$ journal and benefit from:}

- Convenient online submission

- Rigorous peer review

- Immediate publication on acceptance

- Open access: articles freely available online

- High visibility within the field

- Retaining the copyright to your article

Submit your next manuscript at $\gg$ springeropen.com 